\title{
Black Hole Masses and Unification of Seyferts
}

\author{
R. Bachev, G. T. Petrov, L. Slavcheva and B. Mihov \\ Institute of Astronomy, Sofia, Bulgaria, e-mail: bachevr@astro.bas.bg
}

The most commonly invoked power source of Active Galactic Nuclei (AGN) is accretion of galactic gas (probably through a disk) onto a supermassive black hole in the center of the nucleus (Rees 1984). As is well known, a black hole is completely defined by its mass and angular momentum. The unification scheme of active galaxies assumes that two known Seyfert types (Sy1 and Sy2) are not intrinsically different, i. e. their black hole masses, accretion rates and the whole internal structures are identical (Antonucci 1993) and observed differences are due just to a different orientation to the observer of the axisymmetrical central structure (central engine, BLR and thick torus, shadowing broad lines from some directions).

To test this unification scheme we derived black hole masses for 143 Seyfert galaxies using an emission-line (or dynamical) method. This method is based on the assumption that the emitting gas is gravitationally bound, then the FWHM of some line defines the Keplerian velocity (u) of emitting clouds, whose average distance $(R)$ from the center could be approximately estimated from the line luminosity (L), gas density and covering factor. Ionisation equilibrium leads to: $L \_L I N E R^{2} J N n$, where $J$ is the line emissivity, $\omega$ is the covering factor of the region and $\mathrm{N}$ column density. Velocity and distance of the gravitationally bound clouds determine the central mass $(\mathrm{M}): u$ FWHM $(G M / R)^{0.5}$. This central mass is probably dominated by the black hole mass ( $M \mathrm{BH}$ ???) or is proportional to it at least. The [OIII] 5007 narrow line is used (data from Whittle 1992, Nelson \& Whittle 1995), as it is believed the narrow line regions are identical in all Seyfert types. Using that typically $\mathrm{n}=10^{4-5} \mathrm{~cm}^{3}, \mathrm{~N}=10^{20-21} \mathrm{~cm}-2, \mathrm{w}=0.1-0.01$

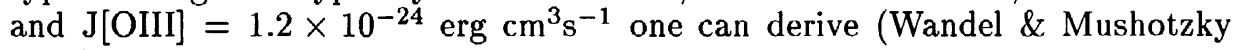
1986):

$$
\log (\mathrm{MBH})=0.5 \log (\mathrm{L}[\mathrm{OIII}])+2 \log (\mathrm{u})-17.7 \text {, }
$$

where MBH is in Solar masses, $\mathrm{L}[\mathrm{OIII}]$ - in $\mathrm{erg} \mathrm{s}^{-1}$ and $\mathrm{u}$ in $\mathrm{km} \mathrm{s}^{-1}$. Most of obtained black hole masses are between $10^{7}$ and $10^{9} \mathrm{M}_{\odot}$. Mass distributions (see the figure) of all types do not show a statistical difference significant enough to separate nuclei into 2 groups based on this parameter, i. e. Seyfert types are indistinguishable through their black hole masses. This result indirectly supports the unified scheme.

Although the sample is not complete, it is interesting to note that Seyfert 2 galaxies with hidden Seyfert 1 nuclei (7 such "hidden Seyfert 1's" are found in the list of AGN used, see for details Antonucci \& Miller 1985) possess the highest masses we derived - about $10^{9} \mathrm{M}_{\odot}$. Thus, these objects fill the high mass tail of the Seyfert 2's distribution. By their masses, they could be well distinguished from both Seyfert 1 and Seyfert 2 galaxies, probably forming a separate subclass. Of course, another possible explanation is the narrow line clouds of these objects 


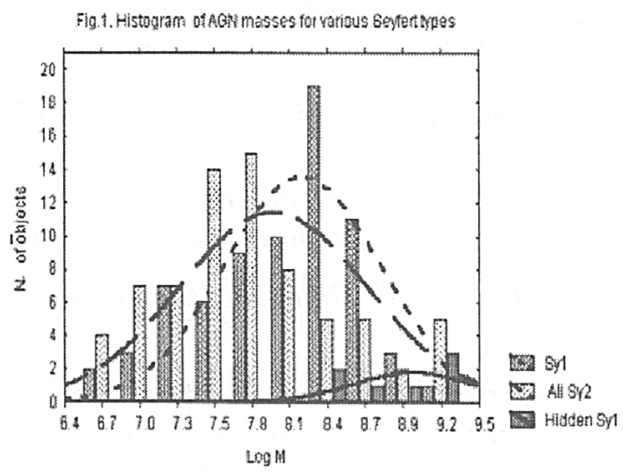

Figure 1. Distributions of black hole masses of Seyfert 1,2 and "Hidden 1 " types. Parameters of normal fits applied to the data are respectively 8.20.6, 8.00.7 and 9.00.4.

are nongravitationally accelerated to velocities quite above the Keplerian one, which may lead to significant overestimation of the mass of the central object. In this case, the emission-line method is inapplicable for the mass estimation. Radio luminosities of these hidden Seyfert 1's are unusually high as well (Moran \& Halpern 1992). Anyway, it seems that these objects are not ordinary Seyfert galaxies and their place in the unified scheme could be quite special.

\section{References}

Antonucci R.R.J.,1993, ARA\&A, [31], 473

Antonucci R.R.J.\& J.S. Miller, 1985, Ap.J., [297], 621

Bachev R. \& Petrov G. T., 1998, to be published in C. R. l'Acad. Bulg. Sci., $[7 / 8]$.

Nelson C. \& M. Whittle, 1995, Ap.J.S.S., [99], No.1

Moran E. C. \& Halpern J. P, in "The Nature Of Compact Objects in AGN", eds. Robinson A., Terlevich R, Cambridge, 1992

Rees M, 1984, ARA\&A,[22],471

Wandel A. \& R.F. Mushotzky, 1986, Ap.J., [306], L61

Whittle M., 1992, Ap.J.S.S., [79], No.1 